\title{
Magnetostrictive-piezoelectric composite structures for energy harvesting
}

\author{
Thomas Lafont $^{1,3}$, L Gimeno ${ }^{1}$, J Delamare ${ }^{1}$, G A Lebedev ${ }^{1,2}$, \\ D I Zakharov ${ }^{1}$, B Viala ${ }^{2}$, O Cugat ${ }^{1}$, N Galopin ${ }^{1}$, L Garbuio ${ }^{1}$ \\ and $O$ Geoffroy ${ }^{1}$ \\ ${ }^{1}$ Grenoble Electrical Engineering Lab(G2ELAB), CNRS UMR 5269, Grenoble-INP, UJF, Grenoble, \\ France \\ ${ }^{2}$ CEA, LETI, MINATEC Campus, Grenoble, France \\ E-mail: thomas.lafont@g2elab.grenoble-inp.fr
}

\begin{abstract}
In this paper, harvesters coupling magnetostrictive and piezoelectric materials are investigated. The energy conversion of quasi-static magnetic field variations into electricity is detailed.

Experimental results are exposed for two macroscopic demonstrators based on the rotation of a permanent magnet. These composite/hybrid devices use both piezoelectric and magnetostrictive (amorphous $\mathrm{FeSiB}$ ribbon or bulk Terfenol-D) materials. A quasi-static (or ultra-low frequency) harvester is constructed with exploitable output voltage, even in quasi-static mode. Integrated micro-harvesters using sub-micron multilayers of active materials on Si have been built and are currently being characterized.
\end{abstract}

(Some figures may appear in colour only in the online journal)

\section{Introduction}

We report investigations on composite structures for energy harvesting under quasi-static conditions. Many harvesters exploit electromechanical conversion to transform, without contact, mechanical energy into electricity. Several kinds of such converters are already in use, but most of them suffer from low output voltage and poor efficiency issues at a low scale $\left(<1 \mathrm{~cm}^{3}\right)$ [1, 2], especially in a quasi-static mode. In order to outperform those harvesters, we suggest a new kind of converter based on the coupling of piezoelectric and magnetostrictive materials. Such so-called artificial magnetoelectric or multi-ferroic composites have already been studied in actuators [3, 4] or sensors [5], and are only very recently being considered for generators [6].

\section{Theory}

\subsection{Limitations of Faraday's law at low speeds}

Electromechanical conversion taking advantage of magnetic field variations seen by a coil is described by Faraday's law,

\footnotetext{
${ }^{3}$ Author to whom any correspondenc should be addressed.
}

which links the time variation of magnetic flux $\phi$ to the electromotive force $e$ :

$$
e=-\frac{\mathrm{d} \phi}{\mathrm{d} t}
$$

For miniature devices, the magnetic flux $\phi$ is correspondingly small since it is proportional to the coil area [1]. Moreover, the $\mathrm{d} t$ term is very large for quasi-static movements. Thus, in slow motion with small devices the electromotive force tends to zero.

For small dimensions, piezoelectric harvesters are used essentially in the vibration mode. This implies a fast and regular mechanical motion. In contrast, for slow motion and small dimensions, our approach is based on the complementary coupling of piezoelectric and magnetostrictive materials.

\subsection{Coupling magnetostriction and piezoelectricity}

Piezoelectric materials are not time dependent if charge leakages are neglected, and thus they may prove useful for harvesting energy from quasi-static (or ultra-low-frequency) events. However, in order to produce electrical energy piezoelectrics require high stress over small displacements, provided through contact forces. 


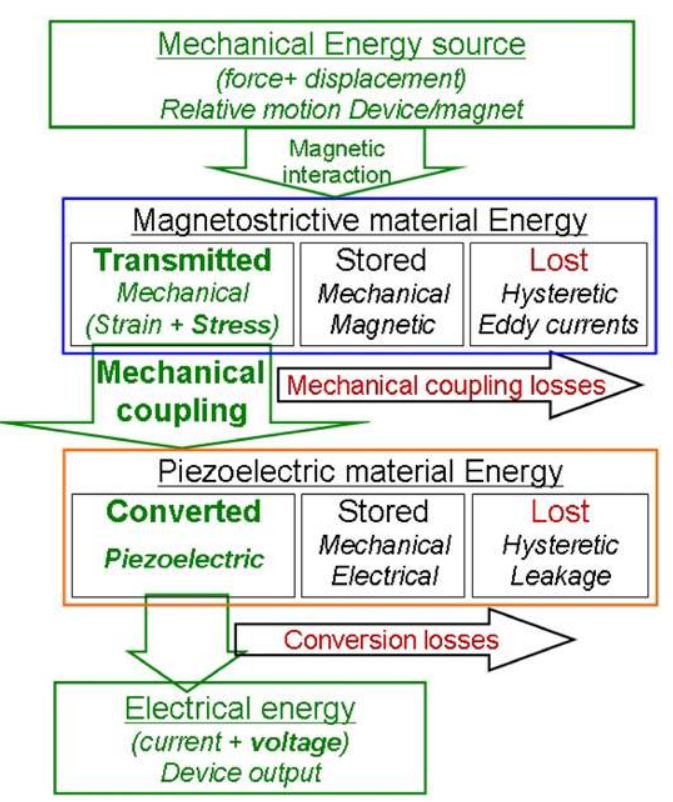

Figure 1. Electromechanical energy conversion.

Magnetostrictive materials are complementary: they can produce high stress to deform a piezoelectric over small strains. Also, they can be 'activated' remotely, via magnetic field noncontact interactions.

In a variable magnetic field, a magnetostrictive material stretches or shrinks. When coupled to a piezoelectric, this deformation displaces electric charges within the material, thus achieving electromechanical conversion. Figure 1 details the various conversion stages.

The coupling of these two materials allows energy conversion from magnetic to mechanical to electricity. In practice, the quasi-static motion of a permanent magnet near the magnetostrictive/piezoelectric hybrid element can produce a relatively high voltage at the output of the piezoelectric element, depending on the device's scale and materials used.

\section{Material choice}

\subsection{Piezoelectric properties}

Piezoelectric materials displace electric charges under mechanical deformation, and vice versa. At present, PMN-PT (lead magnesium niobate-lead titanate) single crystals exhibit the best coupling coefficient in $k_{33}$ mode. This coefficient qualifies the efficiency of energy conversion between electric and mechanical. Nevertheless, these monocrystalline materials are very fragile and expensive. Thus, we choose PZT $\left(\mathrm{PbZr}_{x} \mathrm{Ti}_{1-x} \mathrm{O}_{3}\right)$ oxide ceramics, which also have good coupling coefficients. PZTs are widely available and thin films can be produced by the sol-gel method.

PZTs work along several modes, each mode having its own coupling coefficient. If strain $(\lambda)$ and polarization are in the same direction, we define the $k_{33}$ mode when strain is along a rod and the $k_{\mathrm{t}}$ mode when strain is normal to the plane. When strain and polarization are orthogonal, the $k_{31}$ mode is defined in the case of a rod; in the case of a disc with radial strain we the $k_{\mathrm{p}}$ mode is defined as explained in figure 2 .

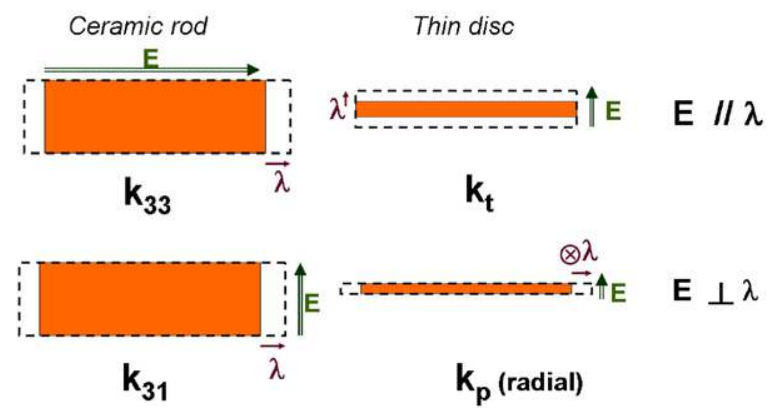

Figure 2. Piezoelectric modes for a rod and a disc.

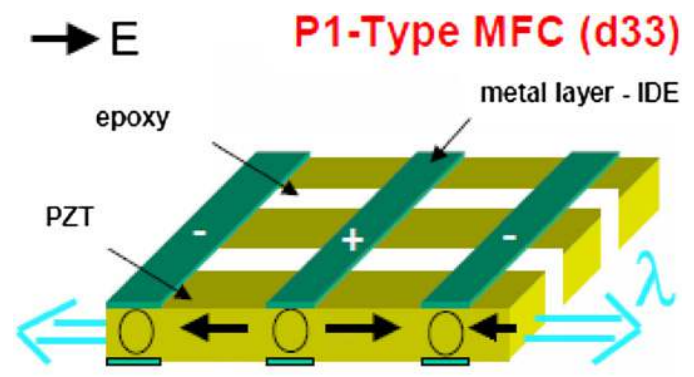

Figure 3. MFC in $k_{33}$ mode [7].

Table 1. Comparison of magnetostrictive materials.

\begin{tabular}{lll}
\hline Material & $\lambda \mathrm{s}(\mathrm{ppm})$ & $\begin{array}{l}\text { Saturation magnetic } \\
\text { field in air A/m (Oe) }\end{array}$ \\
\hline Metglass(FeSiB) & 30 & $400-24000(5-300)$ \\
Terfenol-D & $1500-2000$ & $240000(3000)$ \\
Galfenol & $400-500$ & $24000(300)$ \\
FeCo(bulk) & 80 & $4000(50)$ \\
FeCoB(film) & $30-150$ & $80(1)$ \\
\hline
\end{tabular}

Often, in order to maximize the electric field $E$, piezoelectrics are used in the $k_{31}$ mode. This mode is less effective than the $k_{33}$ mode. The PZT-5 H commercialized by Morgan ceramics [7] exhibits a $k_{31}$ of 0.38 compared to 0.7 for the $k_{33}$ coefficient.

For our devices, we use micro fiber composites (MFC, from Smart Material [8]), made up of PZT-5 microfibers $\left(130 \times 150 \mu \mathrm{m}^{2}\right)$, encased between two Kevlar/Kapton sheets, and polarized along their length. MFCs use PZT in its best mode, and exhibit strains of up to $1500 \mathrm{ppm}$ under $1500 \mathrm{~V}$. Figure 3 presents a schematic view of MFC P1, working in $k_{33}$ mode.

For the complete laminated MFC (Kapton/PZT/Kapton), the coupling coefficient $k_{33}$ is 0.4 . This is better than bulk PZT in $k_{31}$ mode. Moreover, MFCs can easily be coupled to the magnetostrictive material surface.

\subsection{Magnetostrictive properties}

Table 1 compares magnetostrictive materials according to their magnetostriction coefficient $\lambda_{\mathrm{S}}$ and the magnetic induction needed to reach this magnetostriction. $\lambda_{\mathrm{S}}$ qualifies the maximum strain that can be obtained from the material.

Terfenol-D (TbDyFe-based compounds) exhibits the best magnetostriction coefficient but under large magnetic 


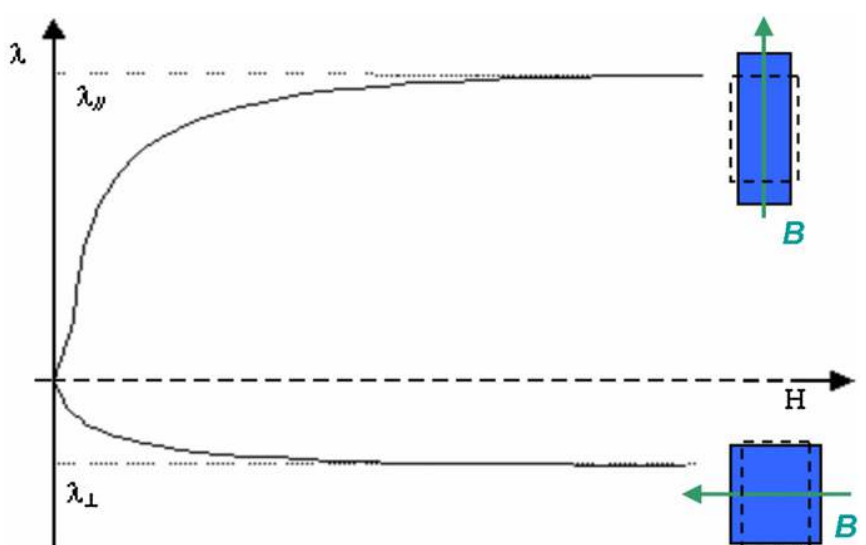

Figure 4. Magnetostrictive materials strain along parallel and perpendicular external magnetic fields.

induction. In contrast, $\mathrm{FeSiB}$ (metglass) has poor magnetostriction, but it has a low saturation magnetic field and is thus very sensitive.

In a magnetostrictive material, the initial orientation of magnetostrictive dipoles is unknown (i.e. when there is no external magnetic field). Figure 4 defines $\lambda_{/ /}$and $\lambda_{\perp}$ for a magnetostrictive material with a positive magnetostriction coefficient, i.e. materials elongating in the direction of the magnetic field. In the case of a material with isotropic behavior, we can define $\lambda_{\mathrm{S}}$ by the following form:

$$
\lambda_{S}=\frac{2}{3}\left(\lambda_{/ /}-\lambda_{\perp}\right) \text {. }
$$

To exploit the full deformation capabilities of the magnetostrictive material, we need to exploit it between its two saturated states $\lambda_{/ /}$and $\lambda_{\perp}$.

The saturated states can be reached in two ways: by a mechanical preload on the magnetostrictive material [9], or by a magnetic field bias. From this initial 'idle' state, a magnetic external field is applied to reach the other state.

\section{Experimental results}

Three piezoelectric-magnetostrictive devices are investigated in this paper. Two macroscopic prototypes are presented first, validating the approach of coupling magnetostrictive and piezoelectric materials in small devices and for slow variations. The operating principle of the conversion is described by the first device; energy conversion and adaptation of the material strain are explored with the second one. Finally, to meet future challenges, a Si-integrated MEMS device is presented.

\subsection{Operating principle}

The first device presents the operating principle of the coupling of piezoelectric and magnetostrictive materials. A simple configuration was chosen in order to underline the principle. The magnetostrictive material was chosen so that no preferential magnetization direction was induced, either by its shape or its properties, thus ensuring that the only magnetic phenomenon in play is magnetostriction.

Consequently, this device is composed of two disks of FeSiB metglass ribbon (diameter $10 \mathrm{~mm}$, thickness $23 \mu \mathrm{m}$ )

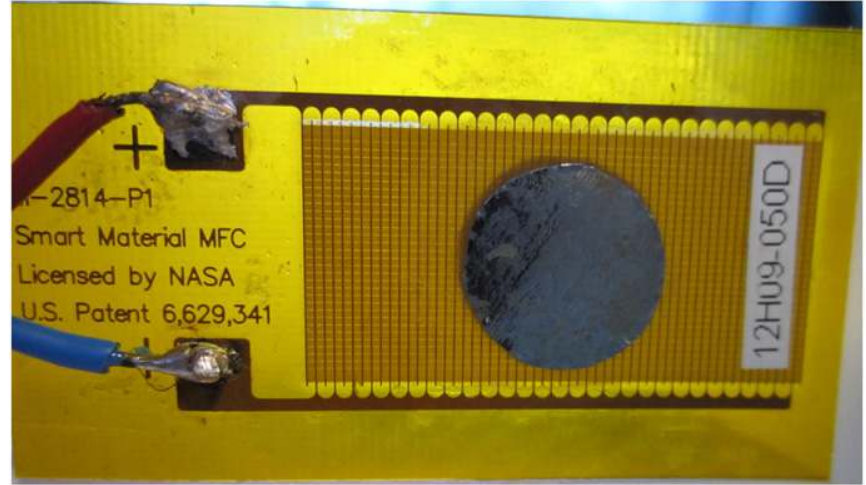

Figure 5. $10 \mathrm{~mm} \mathrm{FeSiB}$ metglass disks glued on both sides of a single MFC.

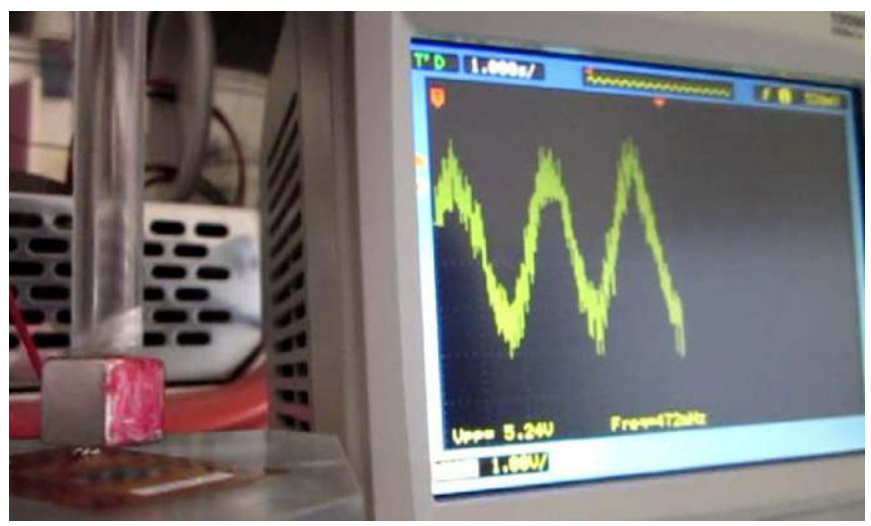

Figure 6. Electric output of device with a permanent magnet rotating slowly (14 rpm) above.

glued on both sides of MFC. FeSiB is amorphous, with full in-plane isotropy. Additionally, the circular geometry of the disks prevents shape anisotropy even if metglass has a huge permeability to concentrate the flux [10] because of the circular geometry. Symmetry to limit bending effects in the laminate is ensured by gluing a metglass disk on each side of the MFC. This first device is presented in figure 5.

When a permanent magnet rotates above the device, the magnetostrictive strain induced in the metglass follows the angular position [2]. The $\mathrm{FeSiB}$ disk state alternates between $\lambda_{/ /}$and $\lambda_{\perp}$ and vice versa, and thus the MFC is stressed by the metglass disk alternately, stretched and shortened twice for one turn of the magnet. When the piezoelectric fibers are stretched, a positive voltage appears between the electrodes and when the piezoelectric material is shortened a negative voltage is observed.

The output of our device in these conditions can be seen in figure 6 . The output voltage is a sinusoïdal signal. The frequency depends on the magnet rotation speed and the amplitude is linked to the magnetic field induced by the permanent magnet, the choice of materials and their mechanical coupling, but is independent of speed to first order. In this case, the voltage amplitude is $5.24 \mathrm{~V}$ for a frequency of $472 \mathrm{mHz}$ for a magnet rotating at $14.2 \mathrm{rpm}$.

Figure 7 shows the evolution of the output voltage with the magnet rotation speed. With this device coupling configuration, the slower the magnet motion, the better 


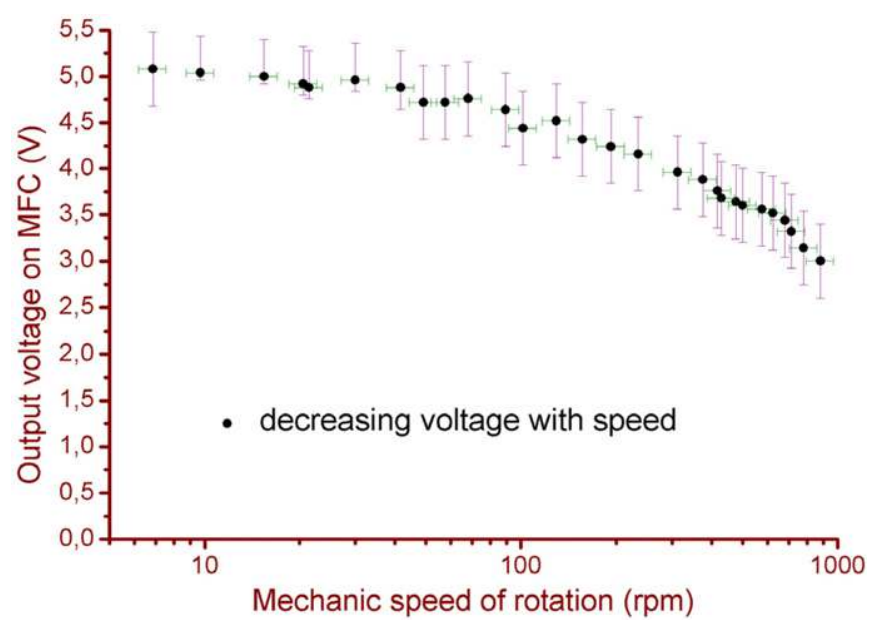

Figure 7. Voltage amplitude as a function of speed of rotation of the magnet.

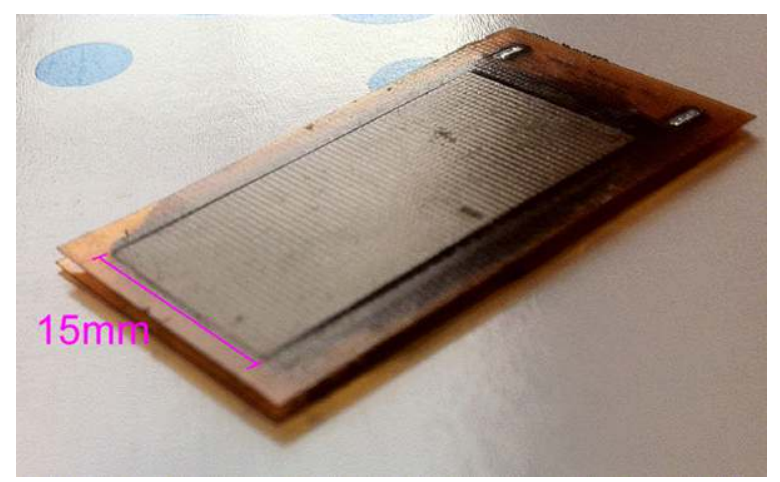

Figure 8. Terfenol-D plate glued between two MFC.

the voltage amplitudes are, thus confirming that this coupling solution for quasi-static harvesting is appropriate. Nevertheless, for very slow rotation speeds, less than $0.3 \mathrm{rpm}$, voltage amplitude will be decreased due to leakage of charges on MFC.

The purpose of this prototype is aimed at exploring the principle, with the choice of materials in terms of mechanical coupling optimal not being. Very thin metglass sheets $\left(\lambda_{\mathrm{S}}=\right.$ $20 \mathrm{ppm}, 23 \mu \mathrm{m}$ thick) deform the MFC only slightly, which can withstand strains up to $1500 \mathrm{ppm}$. Thus, the MFC generates low voltages up to $5 \mathrm{~V}$, whereas it could develop hundreds of volts.

\subsection{Energy measurements}

In order to enhance the strain due to the magnetostriction, the second prototype is made with a $1 \mathrm{~mm}$ thick plate Terfenol$\mathrm{D}$ instead of thin $\mathrm{FeSiB}$ ribbon. The preferred direction is imposed by the coupled geometry and characteristics of the Terfenol-D and MFC elements.

The macroscopic harvester is shown in figure 8 . It is composed of a Terfenol-D plate $\left(30 \times 15 \times 1 \mathrm{~mm}^{3}\right)$ glued between two MFC piezoelectrics. All elements have an active area of $28 \times 14 \mathrm{~mm}^{2}$, thus ensuring a good match between their surfaces. For this configuration, $\lambda_{/ /}$corresponds to the length of the device and $\lambda_{\perp}$ is orthogonal. We choose to use two
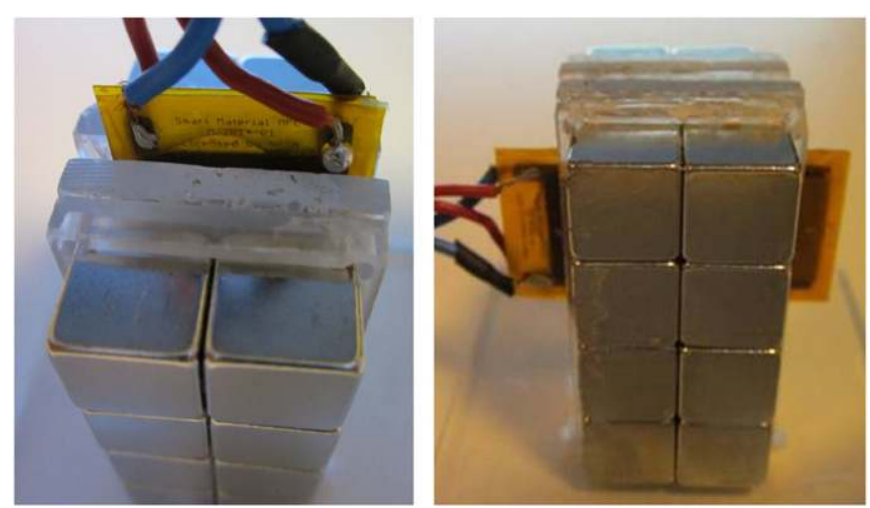

Figure 9. Rotation by $90^{\circ}$ in a permanent magnet magnetic source.

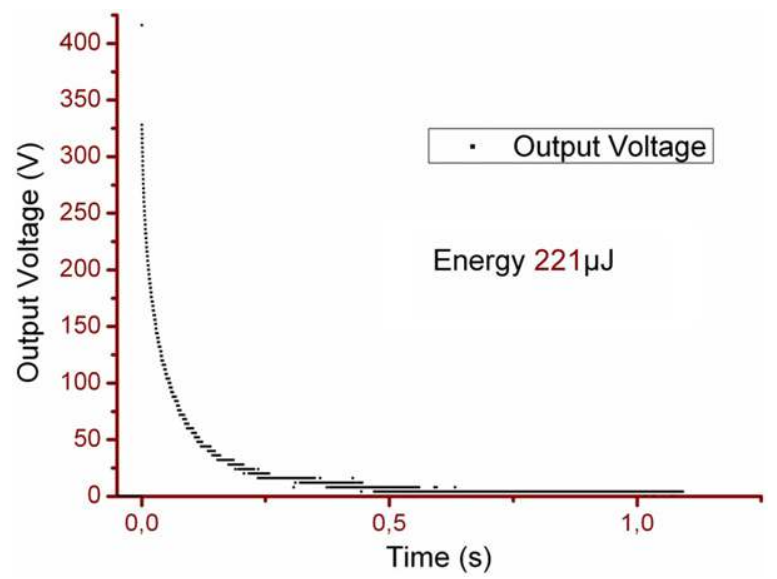

Figure 10. Terfenol/PZT device response to a motion from $\lambda_{/ /}$to $\lambda_{\perp}$. Energy is dissipated in a $10 \mathrm{M} \Omega$ load.

MFCs to keep the symmetry of the device and limit bending effects.

The measurement setup is shown in figure 9. Permanent magnets are used to create a magnetic field vertically between them. To activate the Terfenol-D, it is started in the parallel direction $\left(\lambda_{/ /}\right)$and then rotated by $90^{\circ}$ to arrive at the orthogonal direction $\left(\lambda_{\perp}\right)$.

The energy provided by the MFC is obtained by integrating the voltage measured on the $10 \mathrm{M} \Omega$ load:

$$
E=\int \frac{V^{2}}{R_{\text {Load }}} \mathrm{d} t .
$$

With such a $90^{\circ}$ rotation, even slowly, more than $400 \mathrm{~V}$ is obtained. The energy dissipated in the load is up to $220 \mu \mathrm{J}$ as shown figure 10.

An industrial self-powered wireless switch needs around $100 \mu \mathrm{J}$ to send a 64 bit frame to a base $50 \mathrm{~m}$ away in free air. Thus, with this device configuration, even a slow movement can create enough energy to send a frame to a distant control base, converting enough energy to be used in such an application. It may thus be used as a self-powered wireless sensor, for example, for the detection of opening doors or windows.

4.2.1. Charge leakage. Measurements were done with a contactless voltage probe (infinite input impedance) to gather information about the charge leakage in such a device. 


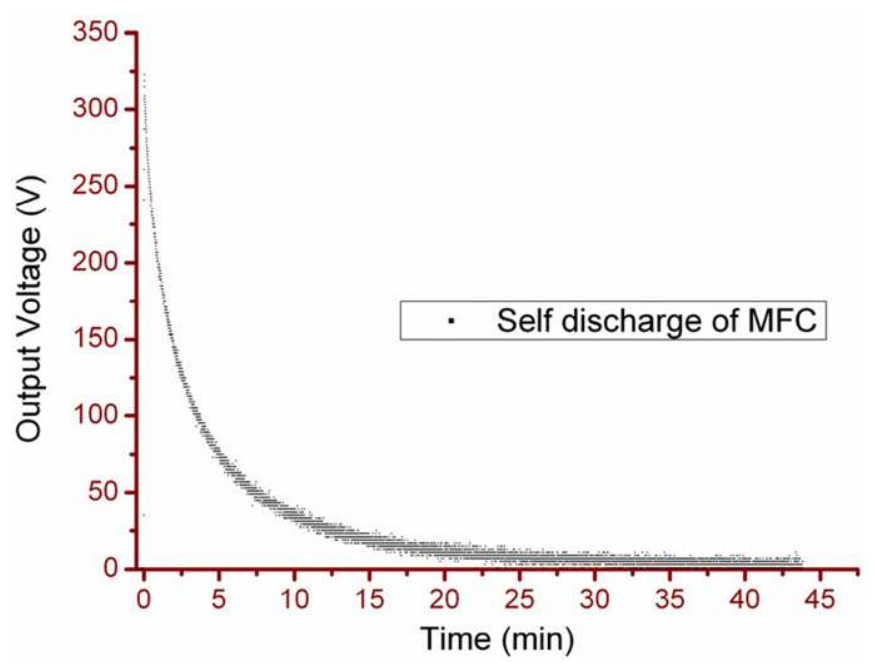

Figure 11. Charge leakage in MFC piezoelectric versus time.

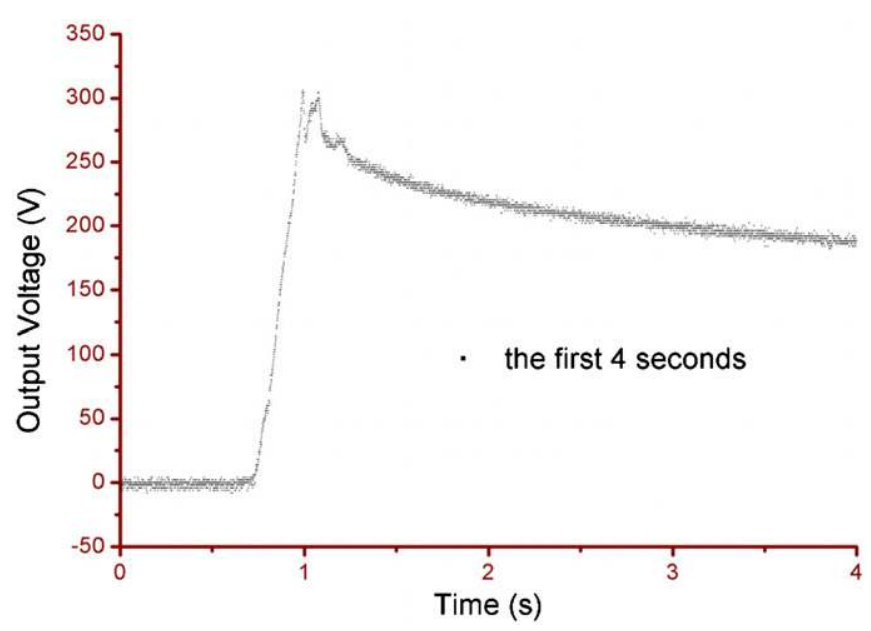

Figure 12. Details of charge leakage, first 4 s.

Figure 11 shows the voltage output measured on the piezoelectric with this voltage probe. The discharge time constant $\tau_{\mathrm{e}}$ due to charge leakage is approximately $5 \mathrm{~min}$. This could be due to the MFC constitution (encapsulated fibers), whereas in bulk ceramics electrical losses seem much larger [11].

Figure 12 represents the first $4 \mathrm{~s}$ of self-discharge. After closing the switch, a fast leakage of charges can be observed in the first second, followed by a slower discharge.

This two-phase discharge seems to point to nonlinearities in the discharge process, which could be due to partial polarization of Kapton. Further work must be performed in order to fully understand the different leakage phenomena and improve the characteristics of such piezoelectrics when used in quasi-static conditions.

\subsection{Integrated device}

The device described in section 4.2 can provide enough energy for existing commercial wireless transmission protocols. In the near future, transmitters based on ultra-wide band technology will appear [12]. The energy required by these chips for low distance transmissions is at present in the order of $100 \mathrm{pJ}$ rather than $100 \mu \mathrm{J}$. With energy requirement forecasts

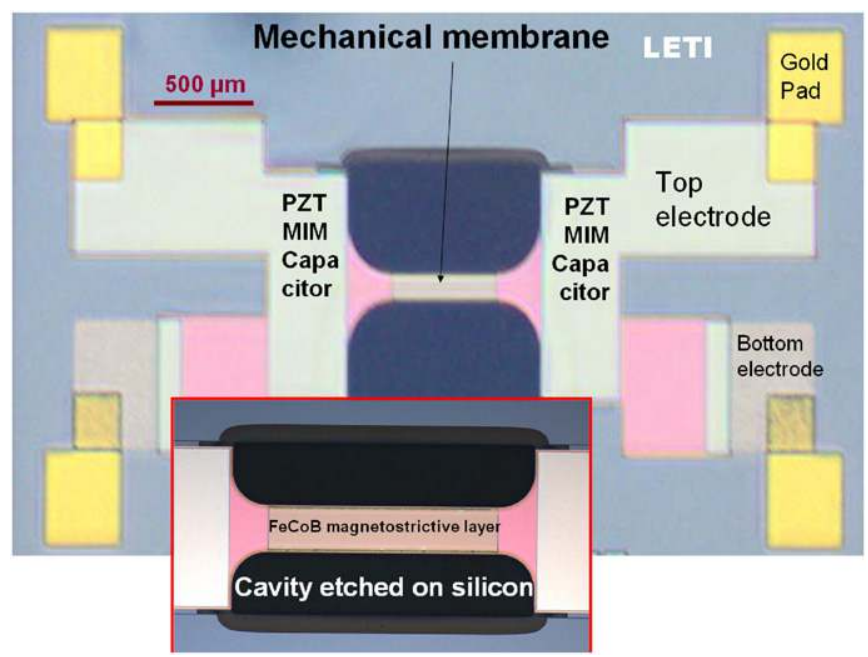

Figure 13. Si-integrated micro-harvester coupling piezoelectric and magnetostrictive sub- $\mu \mathrm{m}$ layers.

being six orders of magnitude smaller than today, we have undertaken the silicon-based integration of our composite magnetostrictive/piezoelectric harvesters.

The piezoelectric layer chosen is sol-gel PZT and the magnetostrictive layer is sputtered FeCoB. Both active material layers are $\sim 1 \mu \mathrm{m}$ thick. The co-integration of both materials (etching of the active layers for pattern definition) and its liberation has been tested and verified, thus validating the fabrication technological process.

Figure 13 shows a Si-based $3 \times 4 \mathrm{~mm}^{2}$ micro-harvester.

The performances of the magnetostrictive layer have been successfully tested separately on a bulk piezoelectric [13] showing magnetic uniaxial anisotropy controlled by E-field with in-plane $d_{33}$ strain mode in the piezo-magnetic composite.

The integrated devices were tested using the piezoelectric/ magnetostrictive coupling in reverse: by applying a voltage to the piezoelectric, the magnetostrictive material is deformed, and the change in magnetization direction is measured.

For the characterization of the harvester mode, the external magnetic field will be provided by a permanent magnet. The mechanical strain produced by the magnetostrictive membrane is transmitted to the piezoelectric and the voltage generated will be measured (tests currently in progress).

\section{Conclusion}

This paper presents our investigation on macroscopic contactless harvesters for slow movements and for small devices. Coupling piezoelectric and magnetostrictive materials allows exploiting quasi-static phenomena. Two types of devices were investigated, both based on MFC/PZT composites.

A simple device, coupling FeSiB and MFC, has been used to demonstrate the principle. It delivers a sinusoidal output voltage when a permanent magnet rotates above, even at sub$\mathrm{Hz}$ speeds. A demonstrator including Terfenol-D has been constructed. A $90^{\circ}$ magnet rotation can produce $220 \mu \mathrm{J}$. This kind of conversion is suitable for low speed displacement.

An integrated micro-harvester has been designed and a first batch of wafers is currently being tested. 


\section{References}

[1] Cugat O, Delamare J and Reyne G 2003 Magnetic micro-actuators and systems (MAGMAS) IEEE Trans. Magn. 39 3607-12

[2] Garbuio L, Lallart M, Guyomar D, Richard C and Audigier D 2009 Mechanical energy harvester with ultra-low threshold rectification based on SSHI non-linear technique IEEE Trans. Ind. Electron. 56 1048-56

[3] Ueno T and Higuchi T 2006 Novel composite of magnetostrictive material and piezoelectric actuator for coil-free magnetic force control Sensor Actuators A 129 251-5

[4] Geoffroy O, O'Brien D, Cugat O and Delamare J 2010 Practical and theoretical investigations of a rotating coil-less actuator using the inverse magnetostrictive effect IEEE Trans. Magn. 46-2 606-9

[5] Huong G D T and Duc N H 2009 Magnetoelectric sensor for microtesla magnetic-fields based on $\left(\mathrm{Fe}_{80} \mathrm{Co}_{20}\right)_{78} \mathrm{Si}_{12} \mathrm{~B}_{10} /$ PZT laminates Sensor Actuator A 149 229-32

[6] Zhang C L, Yang J S and Chen W Q 2009 Harvesting magnetic energy using extensional vibration of laminated magnetoelectric plates Appl. Phys. Lett. 95013511
[7] Berlincourt D and Krueger H H A 2004 Properties of morgan electro ceramics Tech. Publ. 226 TP-232

[8] Smart Material 2010 MFC technical data sheet www.smart-material.com

[9] Yamamoto Y, Eda H and Shimizu J 2002 Application of giant magnetostrictive materials to positioning actuators IEEE/ASME Int. Conf. Advanced Intelligent Mechatronics 1999 pp 215-20

[10] Fang Z, Lu S G, Li F, Datta S, Zhang Q M and El Tahchi M 2009 Enhancing the magnetoelectric response of metglass/polyvinylidene fluoride laminates by exploiting the flux concentration effect Appl. Phys. Lett. 95112903

[11] Zakarov D I et al 2012 Thermal energy conversion by coupled shape memory and piezoelectric effects $J$. Micromech. Microeng. 22094005

[12] Mercier P P, Daly D C and Chandrakasan A P 2009 An energy-efficient all-digital UWB transmitter employing dual capacitively-coupled pulse-shaping drivers IEEE J. Solid State Circuits 44 1679-88

[13] Lebedev G A, Viala B, Lafont T, Zakharov D I, Cugat O and Delamare J 2012 Converse magnetoelectric effect dependence with $\mathrm{CoFeB}$ composition in ferromagnetic/ piezoelectric composites J. Appl. Phys. $11107 \mathrm{C} 725$ 